\title{
Can Human Papillomavirus Testing Replace Papanicolaou Smear in Near Future as a Screening Modality for Cervical Cancers?
}

\author{
Jaideep Malhotra ${ }^{1}$, Ruchika Garg ${ }^{2}$, Pavika Lal ${ }^{3}$ \\ Journal of South Asian Federation of Obstetrics and Gynaecology (2019): 10.5005/jp-journals-10006-1694
}

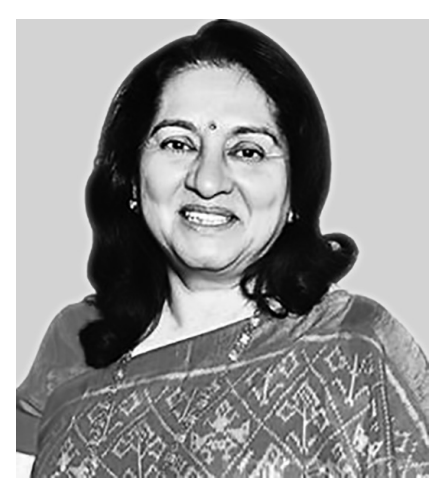

Jaideep Malhotra

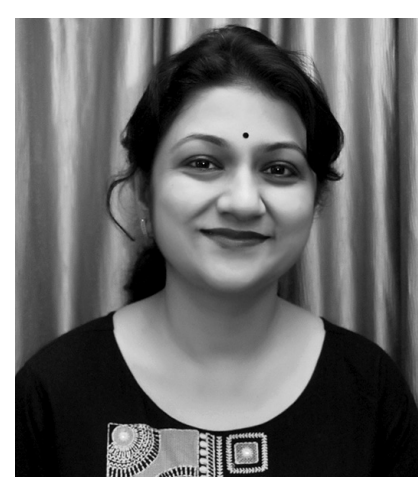

Ruchika Garg

Cervical cancer in Indian women contributes to approximately $6-29 \%$ of all cancers in women ${ }^{1}$ and alone accounts for one-quarter of the worldwide burden of the disease. It is the one of the leading causes of cancer mortality, accounting for $17 \%$ of all cancer deaths among women aged between 30 years and 69 years and occurs in approximately 1 in 53 Indian women during their lifetime compared with 1 in 100 women in more developed regions of the world. ${ }^{2}$ The 5-year relative survival rate for cancer cervix in India has been reported to be approximately $46 \%(34-60),{ }^{3}$ which is much lesser than survival rates reported from other Asian countries.

\section{Screening of Cervical Cancer}

Cervix is amenable to screening by a number of methods which include visual inspection with acetic acid (VIA), magnified VIA (VIAM) visual inspection with Lugol's iodine (VILI), the Papanicolaou (Pap) test, and human papillomavirus (HPV) DNA testing. Pap smear is one of the oldest screening techniques while HPV testing is one of the emerging tests in the screening programs on cervical cancer. In PAP smear, the specificity and sensitivity of liquid-based cytology above conventional cytology is $77.7 \%$ and $55.3 \%$, respectively (while that of conventional is $66.3 \%$ and $51 \%$, respectively), and for HPV, the pooled sensitivity and specificity were $77.81 \%$ and $91.54 \%$, respectively. 4

\section{Limitations of Pap Smear}

The Bethesda system for reporting cervical cell abnormalities ${ }^{6}$ takes into account the various histological subtypes found in the transformation zone of the cervix. Any abnormalities or atypia seen in the cells on the slide hint toward either neoplastic or nonneoplastic changes in the cells. Conventional cytology screening programs have shown a marked decline in the incidence of cervical cancer. However, its successful implementation requires

${ }^{1}$ Rainbow Hospitals, Sikandra, Agra, Uttar Pradesh, India

${ }^{2}$ Department of Obstetrics and Gynaecology, Sarojini Naidu Medical College, Agra, Uttar Pradesh, India

${ }^{3}$ Department of Obstetrics and Gynaecology, Ganesh Shankar Vidyarthi Memorial Medical College, Kanpur, Uttar Pradesh, India

Corresponding Author: Pavika Lal, Department of Obstetrics and Gynaecology, Ganesh Shankar Vidyarthi Memorial Medical College, Kanpur, Uttar Pradesh, India, e-mail: lalpavika@gmail.com

How to cite this article: Malhotra J, Agra R, Garg R, et al. Can Human Papillomavirus Testing Replace Papanicolaou Smear in Near Future as a Screening Modality for Cervical Cancers? J South Asian Feder Obst Gynae 2019;11(3):141-143.

Source of support: Nil

Conflict of interest: None

a variety of requirements to be fulfilled, which are not feasible in many low-resource settings where a high risk of cervical cancer is experienced.

- Cytology screening requires a laboratory infrastructure, microscopes, several resource personnel (smear collectors, cytotechnicians, and pathologists), consumables (slides, fixative, Pap stain containing five dyes, and three solutions), and several steps with inbuilt quality assurance procedures.

- Cytology must be repeated at a frequent rate of 3-5-year intervals to ensure satisfactory sensitivity and optimum detection of cervical cancer precursor lesions, and repeat visits are necessary after a positive cytology for diagnosis and treatment, which may lead to dropouts.

- Several years of cytology screening in low- and middle-income countries have not led to significant reductions in cervical cancer in these countries, possibly due to the difficulties in offering high-quality cytology, programmatic deficiencies in follow-up and treatment of screen-positive women, and also due to the considerable financial, technical, and logistic inputs necessary for effective cytology programs. ${ }^{7,8}$

- Inadequate samples constitute about $8 \%$ of specimens received.

- False-negative rates as high as $20-30 \%$ have been reported. False-negative results can occur from clumping of cells when the cells are not spread evenly and uniformly on the microscope slide.

- Sometimes, other contents of the cervical specimen such as blood, bacteria, or yeast contaminate the sample and prevent the detection of abnormal cells. If exposed to air too long before being fixed on the slide, cervical cells can become distorted.

o The Author(s). 2019 Open Access This article is distributed under the terms of the Creative Commons Attribution 4.0 International License (https://creativecommons. org/licenses/by-nc/4.0/), which permits unrestricted use, distribution, and non-commercial reproduction in any medium, provided you give appropriate credit to the original author(s) and the source, provide a link to the Creative Commons license, and indicate if changes were made. The Creative Commons Public Domain Dedication waiver (http://creativecommons.org/publicdomain/zero/1.0/) applies to the data made available in this article, unless otherwise stated. 
- Human error is probably the primary threat to accurate interpretation. The average Pap smear slide contains 50,000$3,00,000$ cells that must be examined. If the sample contains only a few abnormal cells within a crowded background of healthy cells, the abnormal cells can be missed, particularly by overworked readers.

Hence, these challenges have prompted the search for programs based on alternative cervical screening tests, which are implementable in resource-poor settings.

\section{Human Papillomavirus and its Role in Etiopathogenesis of Cervical Cancers ${ }^{9}$}

Human papillomavirus is one of the most common causes of sexually transmitted disease in both men and women worldwide and is thought to be the most common sexually transmitted viral disease. Human papillomavirus is most commonly virus associated with the gynecological cancers especially cervical cancers in women.

Based on their association with cervical cancer and precursor lesions, HPVs can also be grouped to high-risk and low-risk HPV types. Cervical cancers are mainly caused by types 16 and 18; therefore, detection of HPV has been made an important tool for screening the cervical cancers.

Considering that HPV cannot be grown in conventional cell cultures and serological assays have only limited sensitivity, the diagnosis of HPV infection requires the detection of its genome in cellular samples collected from exfoliated cells of the cervix.

Given that HPV is the cause of cervical cancer and that HPV DNA is detected in virtually all cervical cancers, new screening techniques based on HPV DNA testing have raised hopes and expectations for better prevention of the disease. Testing for HPV DNA is one of the most intensively studied alternatives to cervical cytology screening. The role of HPV testing has already been established and its use has gained wide acceptance in certain areas such as the triage of Pap smears with atypical squamous cell changes (ASCUS smears) and follow-up after treatment. ${ }^{10,11}$

\section{Current Screening Tools}

Recent studies over various places all over the world have found out results which hint toward the restricted role of cytology in detecting the precancerous and cancerous conditions of the cervix in women.

At present, the National Healthcare System (NHS) screening program for cervical cancer starts with a smear test. ${ }^{12}$ Women whose smear test results show possible low-grade or borderline changes to cells will have the sample tested for HPV. Those who have low-grade changes and HPV will then be referred for further tests (i.e., a colposcopy).

In 2003, US was the first country to introduce HPV-based screening. At that time, Pap smear testing in the US was perceived to be of low quality, so they included HPV testing alongside to ensure better quality screening. Since then, many countries like Mexico, Turkey, Netherlands, Sweden, and Italy have introduced HPV testing as a part of their routine screening program for cervical cancers. If this is successful, the NHS may also switch to initial HPV testing.

Various studies have proved that women who had a negative HPV test at the start of the study were much less likely to develop $\mathrm{CIN} 3+$ within 48 months than women who had a negative smear test at the start of the study. ${ }^{13,14}$ It also proved that the number of referrals for colposcopy is very less as compared to referrals when they were solely based on Pap smear which further lead to an increase in the economical burden because of greater requirement of expertise as well as infrastructure.

\section{Advantages of Human Papillomavirus TESTING}

Human papillomavirus testing is better since it can overcome the limitations encountered in Pap smear and can be more cost-effective as compared to Pap smear because of various reasons as follows:

- Since inter- and intraobserver variations encountered in analyzing the results through cytology alone can be overcome now through HPV screening.

- Inadequacy of samples leading to wrong result, technical errors, and high false-negative results obtained through Pap smear can be overcome by HPV.

- Human papillomavirus testing helps us decide whether the patient needs immediate colposcopy or should be returned to routine follow-up in every 5 years.

A latest research has revealed that if HPV testing is delayed even for a month then the chance to stop 48 extra women from developing cervical cancer will be missed..$^{15}$ It has been calculated that a 1-year delay in implementing HPV screening would lead to loss of 1,595 quality-adjusted life years (QALYs), with a value of $£ 32 \mathrm{~m}$ in estimating the cost per QALY. This huge cost because of delayed introduction will produce shocking results. ${ }^{15}$ As such, it is vital that there are no further delays in the rollout of HPV testing in screening of cervical cancers.

\section{Conclusion}

The results of the study show that primary HPV testing detects cervical neoplasia earlier and more accurately than cytology, and it also showed that more CIN3+ and $\mathrm{CIN} 2+$ cases were detected in the first round while there was a significant reduction in the rate of CIN3+ and CIN2+ 48 months later. The results of the study are encouraging and suggest that HPV testing could work well as a primary test in place of cervical smears.

Girls and young women are now offered vaccination against HPV, but it will take some time before this prevents the majority of cases of cervical cancer. Women should continue to take part in cervical cancer screening, whether they have been vaccinated or not. Thus, HPV testing will soon prove to be the only screening tool in the near future for detecting and reducing the incidence of cervical cancers in developing countries.

\section{References}

1. Ferlay J, Soerjomataram I, Ervik M, et al. http://www.globocan.iarc.fr.

2. http://www.healthmetricsandevaluation.org/publications/ policyreport/challenge-ahead-progress-and-setbacksbreastandcervical-cancer.

3. Sankaranarayanan R, Swaminathan R, Lucas E. Cancer Survival in Africa, Asia, Caribbean and Central America: Survcan. Lyon: IARC Scientific Publication International Agency for Research on Cancer; 2010.

4. Bobdey S, Sathwara J, Jain A, et al. Burden of cervical cancer and role of screening in India. Indian J Med Paediatr Oncol 2016;37(4):278-285. DOI: 10.4103/0971-5851.195751.

5. Karimi-Zarchi M, Peighmbari F, Karimi N, et al. A comparison of 3 ways of conventional Pap smear, liquid-based cytology and colposcopy vs cervical biopsy for early diagnosis of premalignant lesions or cervical cancer in women with abnormal conventional Pap test. Int J Biomed Sci 2013;9(4):205-210. 
6. Nayar R, Solomon D. Second edition of 'The Bethesda System for reporting cervical cytology' - Atlas, website, and Bethesda interobserver reproducibility project. CytoJournal 2004;1(1):4. DOI: 10.1186/1742-6413-1-4.

7. Deodhar K, Sankaranarayanan R, Jayant K, et al. Accuracy of concurrent visual and cytology screening in detecting cervical cancer precursors in rural India. Int J Cancer 2012;131(6):E954-E962. DOI: 10.1002/ijc. 27633.

8. Sankaranarayanan R. Screening for cervical and oral cancers in India is feasible and effective. Natl Med J India 2005;18(6):281-284.

9. zur Hausen H. Papillomaviruses in human cancers. Proc Assoc Am Physicians 1999;111(6):581-587. DOI: 10.1046/j.1525-1381.1999. 99723.x.

10. Arbyn M, Buntinx F, Van Ranst M, et al. Virologic versus cytologic triage of women with equivocal Pap smears: a meta-analysis of the accuracy to detect high-grade intraepithelial neoplasia. J Natl Cancer Inst 2004;96(4):280-293. DOI: 10.1093/jnci/djh037.

11. Arbyn M, Sasieni P, Meijer CJ, et al. Chapter 9: clinical applications of HPV testing: a summary of meta-analyses. Vaccine 2006;24 (Suppl 3):S3/78-S3/89. DOI: 10.1016/j.vaccine.2006.05.117.

12. The current NHS cervical cancer screening programme (Analysis by Bazian, Edited by NHS Website).

13. Ogilive GS, van Niekerk D, Krajden $M$, et al. Effect of screening with primary cervical HPV testing vs cytology testing on high-grade cervical intraepithelial neoplasia at 48 months - the HPV FOCAL randomized clinical trial. JAMA 2018;320(1):43-52. DOI: 10.1001/jama.2018.7464.

14. HPV Test May Replace Pap for Some Women By Serena Gordon, HealthDay Reporter, Tuesday, Aug. 21, 2018 (HealthDay News).

15. Delay in replacing the Pap smear with HPV screening is costing lives by Alejandra Castanon, The Conversation. 